\title{
Topologically trapped vortex molecules in Bose-Einstein condensates
}

\author{
R. Geurts, ${ }^{1}$ M. V. Milošević, ${ }^{1,2}$ and F. M. Peeters ${ }^{1, *}$ \\ ${ }^{1}$ Departement Fysica, Universiteit Antwerpen, Groenenborgerlaan 171, B-2020 Antwerpen, Belgium \\ ${ }^{2}$ Department of Physics, University of Bath, Claverton Down, Bath, BA2 7AY, United Kingdom
}

(Received 3 April 2008; published 5 November 2008)

\begin{abstract}
In a numerical experiment based on Gross-Pitaevskii formalism, we demonstrate unique topological quantum coherence in optically trapped Bose-Einstein condensates (BECs). Exploring the fact that vortices in a rotating BEC can be pinned by a geometric arrangement of laser beams, we show the parameter range in which vortex-antivortex molecules or multiquantum vortices are formed as a consequence of the optically imposed symmetry. Being low-energy states, we discuss the conditions for spontaneous nucleation of these unique molecules and their direct experimental observation, and provoke the potential use of the phase print of an antivortex or a multiquantum vortex when realized in unconventional circumstances.
\end{abstract}

DOI: 10.1103/PhysRevA.78.053610

PACS number(s): 03.75.Lm, 47.32.cd, 67.85.Hj

During the last decade, intense experimental and theoretical activity has induced dramatic achievements in BoseEinstein condensation in trapped alkali-metal gases at ultralow temperatures [1]. The atomic Bose-Einstein condensates (BECs) differ fundamentally from the helium BEC, having significantly nonuniform density, very high low-temperature condensate fraction, and being pure and dilute, so that interactions can be accurately parametrized in terms of the scattering length. As a result, a relatively simple nonlinear Schrödinger equation [the Gross-Pitaevskii (GP) equation] gives a precise description of the atomic condensates and their dynamics [2].

It is already well-established that the GP equation has rotating solutions with a well-defined angular momentum, defining the vortex states [3]. Vortices as topological singularities in a BEC have been readily observed in experiment [4] and are also found in liquid helium [5] and in superconductors [6]. The exciting new property of atomic BECs is that intrinsic interactions in the system are directly dependent on the mean particle density [7], which gives them a unique degree of freedom for studies of vortex matter. Another interesting aspect of dilute Bose condensates is that their density can be locally suppressed optically. As experimentally shown by Tung et al. [8], vortex lines in a BEC show great affinity towards weak spots created by focused laser beams, which results in a very profound vortex pinning and provides yet another tool for vortex manipulation [9].

Vortices in a BEC are accompanied by the appropriate distribution of the phase $\varphi$ of the order parameter $\psi$, where the number of successive $2 \pi$ changes along the perimeter corresponds to the vorticity of the vortex state, i.e., the topological charge. Therefore, using the known phase distribution, particular vortex states can be predefined in the system by the so-called phase imprint technique [10], where a uniform light pulse projects a designed mask onto the condensate. However, the gradient of the imprinted phase $(\nabla \varphi)$ must always be parallel to the rotational velocity of the condensate, i.e., the antivortex is fundamentally unstable for a positive angular momentum. Note also that imprinted mul-

\footnotetext{
*francois.peeters@ua.ac.be
}

tiple $2 \pi$ change, i.e., a multiquantum vortex, was never found stable against decomposition into single vortices in conventional BEC setups [11].

Motivated by these recent developments in vortex physics, in this paper we exploit a uniquely defined BEC system that combines the above essentials; our atomic BEC is confined, exposed to engineered spatial pinning, and is cylindrically rotated. Knowing that vortex solitons are strongly influenced by symmetry in nonlinear media [12], we impose a particular symmetry on the system by chosen arrangement of the vortex-trapping optical beams. As we will show, this results in the nucleation of vortex molecules of matching symmetry in the ground state, which may comprise unconventional phase profiles containing a stable antivortex, or a multiquantum vortex.

For the purpose of this work, we solve the stationary GP equation in a rotating frame with frequency $\Omega$ around the $z$ axis:

$$
\left(-\frac{\hbar^{2}}{2 m} \Delta+V_{c}+g|\psi|^{2}-\Omega L_{z}-\mu\right) \psi=0,
$$

where $V_{c}$ stands for confinement potential, $g=4 \pi \hbar^{2} a / m$ is the nonlinearity with $a$ being the $s$-wave scattering length, $L_{z}=-i \hbar\left(x \partial_{y}-y \partial_{x}\right)$ is the angular momentum operator, $\mu$ denotes chemical potential, and $\psi$ is the order parameter normalized to the number of particles in the condensate $N$. The energy of a particular state is then given by $E=\mu$ $-\frac{g}{2} \int d V|\psi|^{4}$. We take the usual parabolic-like confinement potential characterized by the frequency $\omega_{\perp}$ in the $(x, y)$ plane and $\omega_{z}$ in the $z$ direction. Our numerical method treats the GP equation in all three dimensions, but in the present work we take $\omega_{z} \gg \omega_{\perp}$ forcing the condensate in a quasi-twodimensional, oblate (pancake) shape. In that case, Eq. (1) can be written in dimensionless and discretized form as

$$
\begin{aligned}
& \frac{U_{x}^{i-1, i} \psi_{i-1, j}}{b_{x}^{2}}+\frac{U_{x}^{i+1, i} \psi_{i+1, j}}{b_{x}^{2}}+\frac{U_{y}^{j-1, j} \psi_{i, j-1}}{b_{y}^{2}}+\frac{U_{y}^{j+1, j} \psi_{i, j+1}}{b_{y}^{2}} \\
& =\left(\frac{2}{b_{x}^{2}}+\frac{2}{b_{y}^{2}}-g^{\prime}\left|\psi_{i, j}\right|^{2}-V+\mu\right) \psi_{i, j},
\end{aligned}
$$

where $b_{x, y}$ are the lattice constants of the Cartesian grid, and 
the link variable is defined as $U_{\alpha=x, y}^{m, n}=\exp \left[-i \int_{\mathbf{r}_{m}}^{\mathbf{r}_{n}} \mathbf{A}_{\alpha}(\mathbf{r}) d \alpha\right]$, with $A_{x(y)}=(-) \frac{1}{2} \Omega y(x)$. The distances, angular velocity, and energy are expressed in the fundamental scales of the harmonic trap, i.e., $r_{0}=\sqrt{\hbar / m \omega_{\perp}}, \omega_{0}=\omega_{\perp}$, and $E_{0}=\hbar \omega_{\perp}$, respectively. The two-dimensional nonlinearity $g^{\prime}$ $=2 a N \sqrt{2 \pi m \omega_{z} / \hbar}$ (found by averaging the GP equation over the $z$ direction) now depends on $N$ since we chose wave function normalized to unity. Therefore, for fixed $g, N$ is a measure of the importance of interactions in the condensate and directly reflects on the vortex phase diagram. Note also that for low nonlinearities, i.e., when $g^{\prime}$ is small, vortex states in parabolic confinement are stable only for velocities of the condensate close to $\Omega=\omega_{\perp}$, i.e., when the gas dissolves due to centrifugal forces. To enhance the vortex stability, we have chosen a modified confinement potential $V_{c}$ $=\frac{1}{2} r_{\perp}^{2}+\frac{1}{10} r_{\perp}^{4}$. Such empowered confinement enables the condensate to survive higher angular velocities $\left(\Omega>\omega_{\perp}\right)$ and is feasible in experiment by combining optical and magnetic trapping (e.g., for ${ }^{87} \mathrm{Rb}$, a magnetic trap with frequency $75.5 \times 2 \pi \mathrm{Hz}$ in combination with a laser beam with waist $25 \mu \mathrm{m}$ and power $1.2 \mathrm{~mW}[13])$.

\section{VORTEX-ANTIVORTEX MOLECULES AS THE GROUND STATE}

In this paper, we consider a ${ }^{23} \mathrm{Na} \mathrm{BEC}$, with somewhat lower interaction than ${ }^{87} \mathrm{Rb}$, and consequently lower nonlinearity. With the above expression for $V_{c}$, we use the effective perpendicular parabolic confinement with $\omega_{\perp}=4.39$ $\times 2 \pi \mathrm{Hz}$, and the unit of length becomes $r_{0}=10 \mu \mathrm{m}$. To obtain the pancake shape of the condensate, we take $\omega_{z}$ $=10 \omega_{\perp}$. The nonlinearity parameter for a condensate consisting of $10^{4}$ sodium atoms becomes $g^{\prime}=44$. In what follows, we implement laser pinning in the formalism and model it as a Gaussian potential well $V_{p}=\exp \left[-16\left(r / w_{p}\right)^{2}\right](r$ is the distance from the pinning center) as depicted schematically in the inset of Fig. 1(c). We use a set of $N_{p}$ laser beams on a single ring, and compare first the energy in the absence and presence of pinning, as shown in Fig. 1(c). The resulting total potential in the BEC, introduced by confinement and laser beams, is taken as

$$
V=V_{c}(\vec{r})+a_{p} \sum_{j=1, N_{p}} V_{p}\left(\vec{r}-\vec{r}_{j}\right),
$$

with $\vec{r}_{j}=d_{p}\left[\cos \left(j 2 \pi / N_{p}\right) \vec{e}_{x}+\sin \left(j 2 \pi / N_{p}\right) \vec{e}_{y}\right]$. With increasing angular velocity $\omega=\Omega / \omega_{\perp}$, vortices stabilize in the system, and each vortex state exhibits a distinct energy. In the $N_{p}$ $=0$ case, for chosen parameters, individual vortices form multivortex patterns denoted by $\left(L_{c}, L\right)$, where $L_{c}$ gives the number of vortices in the central part of the condensate, i.e., $L_{c}=\lim _{\epsilon \rightarrow 0} \frac{i}{2 \pi \epsilon} \oint_{|\vec{r}|=\epsilon} d\left(\log \frac{\psi}{\psi \psi}\right)$, and $L$ is the total vorticity. In the $N_{p} \neq 0$ case, the ordering of laser beams imposes its symmetry on the vortex states, with $N_{p}$ vortices pinned by the laser beams, and the remaining ones sitting in the central region of the condensate. As a remarkable phenomenon, the system may preserve the symmetry even for $L<N_{p}$; e.g., for $L=N_{p}-1$, still $N_{p}$ vortices nucleate and are pinned, but must be accompanied by a central antivortex in order to simulta-

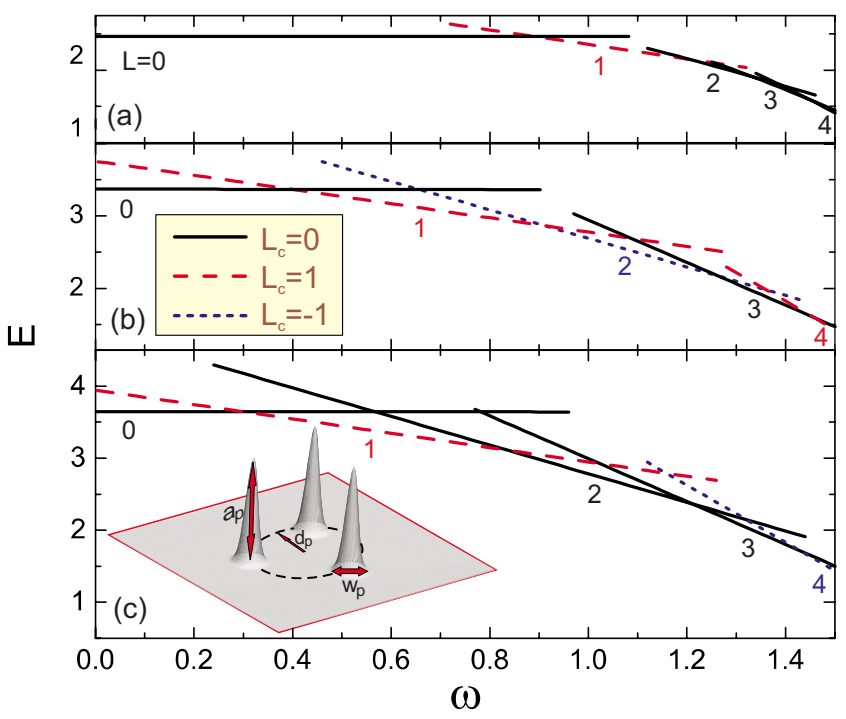

FIG. 1. (Color online) Energy versus angular velocity of a ${ }^{23} \mathrm{Na}$ condensate with $g^{\prime}=20$ (i.e., with $N \approx 5000$ atoms), in the case of no laser pinning $\left(N_{p}=0\right)(\mathrm{a})$, and three $\left(N_{p}=3\right)$ (b), and five $\left(N_{p}\right.$ $=5$ ) (c) laser beams in a polygonal setting (with $d_{p}=0.5, w_{p}=0.4$, $a_{p}=500$ ). Different lines correspond to states with different total vorticity $L$. The colors and style of the lines indicate the vorticity in the center of the condensate $\left(L_{c}\right)$. The inset depicts the used energy profile for each pinning site and notation of the parameters.

neously match the total angular momentum and the symmetry of the pinning potential. Energy levels of such vortexantivortex molecules are indicated in Fig. 1 for $N_{p}=3$ and 5 by the dotted (blue) curves.

Vortex-antivortex (V-AV) phenomena has already been of interest in a BEC, mainly due to the fact that the excitation energy of a vortex-antivortex pair in nonrotating condensate is lower than the one of a single vortex. For that reason, it was assumed that the actual nucleation of a vortex occurs through the generation of a V-AV pair and the subsequent expulsion of the antivortex [14]. While this may hold in an ideally noninteracting BEC, V-AV appearance is seriously hampered by even small perturbations in the system, particularly in the rotating one. Yet, Crasovan et al. demonstrated that a V-AV pair may be stable in an interacting nonrotating BEC, however, only as an excited state (ground state remains vortex-free) [15] and the dynamics of such a state was recently analyzed in Ref. [16]. In addition, vortex-antivortex clusters were studied in Ref. [17], the stabilization of vortexantivortex lattices was considered in Ref. [18], and a superposed vortex-antivortex state was investigated in Ref. [19], however, all only for the case of nonrotating systems. Here we show that states comprising an antivortex can be stabilized as the ground state in a rotating BEC by engineered pinning. Moreover, by using a different number of laser beams, antivortex can be realized at different angular velocities. Figure 2 shows the $\omega$-stability range of $\mathrm{V}$-AV states with one antivortex found for $N_{p}=3-7$, thus all states having vorticity $L=N_{p}-1$. With increasing $N_{p}$, not only V-AV states appear for higher angular momenta, but they can also be spread over a larger area of the condensate. When artificially spreading the laser beams further apart (increasing $d_{p}$, see 


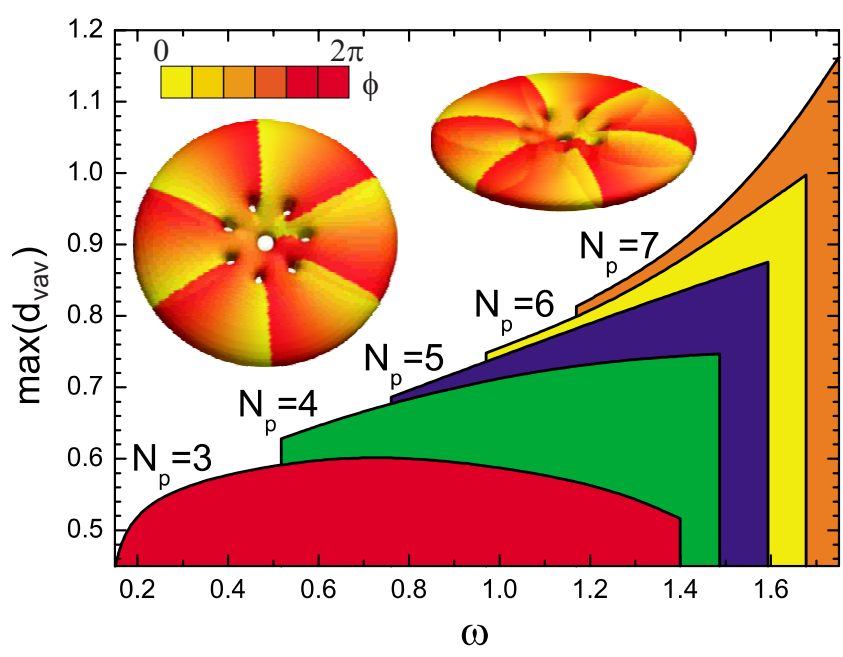

FIG. 2. (Color online) Histogram of vortex-antivortex (V-AV) distance in stable molecules found for a different number of equidistant laser beams on a ring, as a function of angular velocity for $g^{\prime}=40$. Maximal V-AV separation increases for more used pinning beams, and insets show two oblique views of the superimposed density or phase three-dimensional plots of the condensate for $\omega$ $=1.71$ and $N_{p}=7$.

Fig. 1), vortices spontaneously follow and increase their distance from the central antivortex $\left(d_{\text {vav }}\right)$ which facilitates the experimental verification of the $\mathrm{V}-\mathrm{AV}$ molecule. Figure 2 also shows the maximal $d_{v a v}$ that can be reached by increasing $d_{p}$ for $w_{p}=0.5, a_{p}=500$, and $g^{\prime}=40$. The insets of Fig. 2 show isosurfaces of density $10^{-6}$ on which the phase distribution is superimposed for a condensate in the $\mathrm{V}$-AV state for $N_{p}=7, g^{\prime}=22$, and $\omega=1.71$.

\section{SPECIAL PHASE TRANSITIONS}

As much as V-AV states are exciting study objects, our system actually exhibits even richer vortex structures beyond $\mathrm{V}$-AV phenomena. To illustrate this, we constructed a full vortex phase diagram, shown in Fig. 3 as a function of $\omega$ and nonlinearity $g^{\prime}$, for $N_{p}=4$, i.e., a square arrangement of laser beams. As shown before, for constant $g^{\prime}$, the vorticity increases with increasing $\omega$. Note, however, that our strategic pinning setup enforces the $N_{p}$-fold symmetry of the vortex states, which leads to specific transitions in the central region of the condensate. As illustrated by a color gradient in Fig. 3, the central vorticity changes as $L_{c}=\bmod \left(L+1, N_{p}\right)-1$, where an antivortex may nucleate as discussed above. Nevertheless, $N_{p}$-fold symmetry tends to remain preserved for other vorticities as well, which results in the compression of excess $L-k N_{p}\left(k \in \mathbb{N}^{+}\right)$vortices in the center of the condensate into a multiquantum vortex (also called giant vortex). This result complements earlier studies of such vortices in conventional BEC setups, where a multiquanta vortex was found unstable towards decay into single-quantum vortices [11]. For $N_{p}=4$, we show the $0,1,2,-1,0,1,2,-1$ sequence with increasing $\omega$ in Fig. 3, for total vorticity $L=0-7$. A doubly quantized vortex was realized for $L=2$ and $L=6$ states in the center of the condensate.

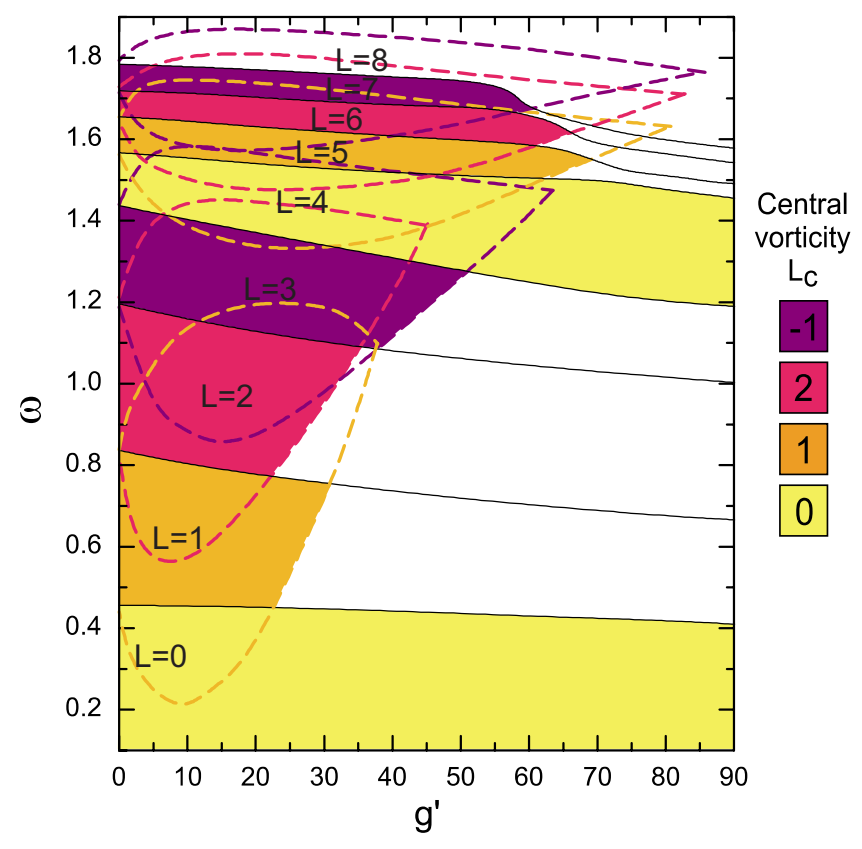

FIG. 3. (Color online) Equilibrium vortex phase diagram as a function of the angular velocity and nonlinearity of the condensate, for $N_{p}=4, d_{p}=0.71, w_{p}=0.4$, and $a_{p}=500$. Coloring scheme illuminates the vorticity in the center of the condensate, between the pinning beams. Solid lines show the transitions in the ground state, while dashed curves illustrate the whole region of stability for different vortex states. In white areas, the vortex structure does not fully obey the fourfold symmetry of the pinning.

While the existence of stable multiquantum vortices was already predicted in several theoretical papers for condensates in a confinement potential with a quartic component [20], our giant vortices are solely a consequence of the symmetry induced by the pinning centers, as can be deduced from Fig. 4(d) for the $L=6=4+2$ state: The giant vortex state is not stable when $w_{p}$ is too small. By doing two separate simulations of a condensate in conventional parabolic confinement - thus without quartic component-exposed to four pinning beams, we were able to confirm this statement: We found a giant vortex in both the ground states for $L=2$ (with $g=20, \omega=0.85, d_{p}=0.8, w_{p}=0.6, a_{p}=50$ ) and $L=6=4$ $+2\left(g=40, \omega=0.85, d_{p}=1.3, w_{p}=1.6, a_{p}=200\right)$. This general behavior holds for an arbitrary number of pinning beams. In other words, vortex molecules comprising multiquantum vortices are stable in our system, contrary to conventional behavior of BEC. Moreover, we report here a unique way of engineering locally the phase imprint of the condensate, by either changing angular velocity, or the nonlinearity (e.g., by condensing more constituent atoms). The limits of this procedure are (i) the deconfinement of the condensate at large $\omega$, and (ii) the maximal $g^{\prime}$ at which vortex-vortex interaction overwhelms the optical pinning, resulting in a vortex structure that does not fully obey the imposed geometry (white areas in Fig. 3).

\section{STABILITY AND CONTROL OF SPECIAL VORTEX STATES}

Obviously, the exact parameters of the strategically placed laser beams are very deterministic for the resulting vortex 


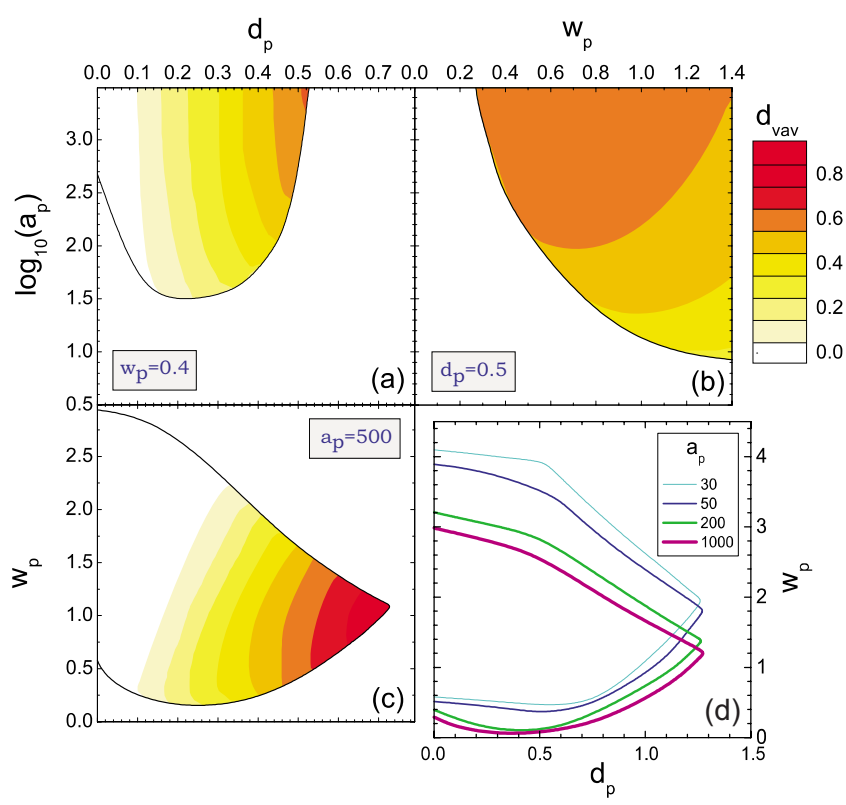

FIG. 4. (Color online) Influence of the optical parameters of the laser beams on the stability of the special vortex molecules found for $N_{p}=4$. (a)-(c) V-AV stability and spacing in a two-parameter space, thus for either fixed amplitude, separation, or focused width of the beams $\left(g^{\prime}=40, \omega=1.25\right)$. (d) The stability region of the doubly quantized vortex in the center of a $L=6$ state, for different values of $a_{p}\left(g^{\prime}=40, \omega=1.6\right)$.

structure in the condensate. Here we briefly discuss the influence of beam intensity, distance between adjacent beams, and their focused waist on V-AV and multiquantum states. Our results are summoned in Fig. 4. Figures 4(a)-4(c) relate to $\mathrm{V}$-AV molecules in fourfold pinning and help visualize their stability and size in a two-parameter space. As a general conclusion, we find that a larger amplitude of the pinning $\left(a_{p}\right.$, see inset of Fig. 1) is always beneficial to the V-AV state. Simply, stronger imposed pinning reflects better its symmetry on the vortex state. For the same reason, strong overlap between pinning profiles of the beams should be avoided (i.e., $w_{p} \lesssim 3 d_{p}$ is desirable). As shown in Fig. 4 , the distance between the central antivortex and pinning vortices can be increased by increasing the spacing between the beams $\left(d_{p}\right)$; this is nevertheless a limited option, as vortices may not follow the imposed pinning at large distances from the center of the condensate. For considered parameters, we realized maximal V-AV distance of approximately $0.9 r_{0}$, which is of the order of $10 \mu \mathrm{m}$ for the sodium condensate, and thus feasible for experimental observation. The used beam width of $w_{p}=0.2-1.5 r_{0}$ and sub- $r_{0}$ distances for beam ordering are also very realistic values for the available experimental techniques.

Figure 4(d) shows the stability region of a doubly quantized vortex in a fourfold geometry of the $L=6$ state $(4 \times 1$ +2 ). Similarly to the V-AV case, imposed symmetry of the pinning is essential for the nucleation of the double vortex. Relatively weaker pinning by wider laser beams can significantly enhance the stability of the multiquantum vortex, while the clarity of its separation from the surrounding vortices is best achieved for high beam amplitudes and narrow beam waists. However, the needed $d_{p}$ is quite low, as confinement between the pinning beams must remain firm for the stabilization of the multiquantum vortex. Such small spacing is unfavorable for experimental observation, but can be significantly improved in the case of larger $N_{p}$ and larger vorticity. This analysis and the complete investigation of all stable vortex states in this phenomenologically rich system will be presented elsewhere.

\section{CONCLUSIONS}

In summary, using a concept of polygonal optical pinning, we demonstrated previously unpredicted vortex states in rotating atomic Bose-Einstein condensates. We realized a stable antivortex for positive angular momentum, as well as a multiquantum vortex, which up to now was assumed to be unstable in a BEC. Both are found as the ground state for a wide range of parameters. Unique ground-state transitions found by increasing angular velocity of the condensate, by changes in the optical pinning setup, or by changing the number of constituent atoms, open up ways to further studies employing quantum phase engineering [10] and submicron coherence and matter-wave interference effects [21].

\section{ACKNOWLEDGMENTS}

The authors thank $J$. Tempère for a critical reading of the paper. This work was supported by the Flemish Science Foundation (FWO-VI). M.V.M. acknowledges support from the EU Marie-Curie Intra-European program. The work was partially done during a visit of F.M.P. to the Institute for Mathematical Sciences, National University of Singapore in 2007.
[1] M. H. Anderson, J. R. Ensher, M. H. Matthews, C. E. Wieman, and E. A. Cornell, Science 269, 198 (1995); K. B. Davis, M.-O. Mewes, M. R. Andrews, N. J. van Druten, D. S. Durfee, D. M. Kurn, and W. Ketterle, Phys. Rev. Lett. 75, 3969 (1995).

[2] E. M. Lifshitz and L. P. Pitaevskii, Statistical Physics (Pergamon, Oxford, 1980), Vol. 2.

[3] G. Baym and C. J. Pethick, Phys. Rev. Lett. 76, 6 (1996).
[4] M. R. Matthews, B. P. Anderson, P. C. Haljan, D. S. Hall, C. E. Wieman, and E. A. Cornell, Phys. Rev. Lett. 83, 2498 (1999); M. W. Zwierlein, J. R. Abo-Shaeer, A. Schirotzek, C. H. Schunck, and W. Ketterle, Nature (London) 435, 1047 (2005).

[5] D. R. Tilley and J. Tilley, Superfluidity and Superconductivity (Hilger, Bristol, 1986).

[6] M. Tinkham, Introduction to Superconductivity (McGraw-Hill, New York, 1996). 
[7] F. Dalfovo, S. Giorgini, L. P. Pitaevskii, and S. Stringari, Rev. Mod. Phys. 71, 463 (1999).

[8] S. Tung, V. Schweikhard, and E. A. Cornell, Phys. Rev. Lett. 97, 240402 (2006).

[9] K. Kasamatsu and M. Tsubota, Phys. Rev. Lett. 97, 240404 (2006).

[10] J. Denschlag, J. E. Simsarian, D. L. Feder, C. W. Clark, L. A. Collins, J. Cubizolles, L. Deng, E. W. Hagley, K. Helmerson, W. P. Reinhardt, S. L. Rolston, B. I. Schneider, and W. D. Philips, Science 287, 97 (2000).

[11] D. A. Butts and D. S. Rokhsar, Nature (London) 397, 327 (1999); A. E. Leanhardt, A. Görlitz, A. P. Chikkatur, D. Kielpinski, Y. Shin, D. E. Pritchard, and W. Ketterle, Phys. Rev. Lett. 89, 190403 (2002).

[12] Y. V. Kartashov, A. Ferrando, A. A. Egorov, and L. Torner, Phys. Rev. Lett. 95, 123902 (2005); V. M. Pérez-García, M. A. García-March, and A. Ferrando, Phys. Rev. A 75, 033618 (2007); M. A. García-March, A. Ferrando, M. Zacarés, J. Vijande, and L. D. Carr, e-print arXiv:0806.0947v1.

[13] V. Bretin, S. Stock, Y. Seurin, and J. Dalibard, Phys. Rev. Lett. 92, 050403 (2004).

[14] C. Raman, J. R. Abo-Shaeer, J. M. Vogels, K. Xu, and W. Ketterle, Phys. Rev. Lett. 87, 210402 (2001); Q. Zhou and H.
Zhai, Phys. Rev. A 70, 043619 (2004).

[15] L.-C. Crasovan, G. Molina-Terriza, J. P. Torres, L. Torner, V. M. Pérez-García, and D. Mihalache, Phys. Rev. E 66, 036612 (2002); L.-C. Crasovan, V. Vekslerchik, V. M. Pérez-García, J. P. Torres, D. Mihalache, and L. Torner, Phys. Rev. A 68, 063609 (2003).

[16] W. Li, M. Haque, and S. Komineas, Phys. Rev. A 77, 053610 (2008)

[17] M. Möttönen, S. M. M. Virtanen, T. Isoshima, and M. M. Salomaa, Phys. Rev. A 71, 033626 (2005); V. Pietilä, M. Möttönen, T. Isoshima, J. A. M. Huhtamäki, and S. M. M. Virtanen, ibid. 74, 023603 (2006).

[18] G. Ruben, D. M. Paganin, and M. J. Morgan, Phys. Rev. A 78, 013631 (2008).

[19] M. Liu, L. H. Wen, H. W. Xiong, and M. S. Zhan, Phys. Rev. A 73, 063620 (2006).

[20] A. Aftalion and I. Danaila, Phys. Rev. A 69, 033608 (2004); I. Danaila, ibid. 72, 013605 (2005); A. L. Fetter, B. Jackson, and S. Stringari, ibid. 71, 013605 (2005); K. Kasamatsu and M. Tsubota, Phys. Rev. Lett. 97, 240404 (2006).

[21] Atom Interferometry, edited by P. R. Berman (Academic, Cambridge, 1997) 